\title{
Novos táxons infraespecíficos e combinações em Pilocarpus Vahl (Rutaceae) $^{1}$
}

\author{
LADISLAU A. SKORUPA ${ }^{2}$
}

(recebido: 2 de maio de 2002; aceito: 23 de abril de 2003)

\begin{abstract}
New infraspecific taxa and combinations in Pilocarpus Vahl (Rutaceae). New infraspecific taxa and combinations in Pilocarpus are presented in anticipation to a complete publication of the taxonomic revision of the genus: $P$. grandiflorus Engl. var. recurvus Skorupa; P. pauciflorus A. St.-Hil. subsp. clavatus Skorupa; P. racemosus Vahl subsp. goudotianus (Tul.) Skorupa; P. racemosus Vahl subsp. goudotianus (Tul.) Skorupa var. mollis (Cuatrec.) Skorupa; P. racemosus Vahl subsp. goudotianus (Tul.) Skorupa var. heterochromus (Kaastra) Skorupa. Data on the geographic distribution, habitat and phenology are given for these taxa.
\end{abstract}

Key words - Pilocarpus, Rutaceae, taxonomy

RESUMO - (Novos táxons infraespecíficos e combinações em Pilocarpus Vahl (Rutaceae). Novos táxons infraespecíficos e combinações em Pilocarpus são apresentados em antecipação à completa publicação da revisão do gênero: P. grandiflorus Engl. var. recurvus Skorupa; P. pauciflorus A. St.-Hil. subsp. clavatus Skorupa; P. racemosus Vahl subsp. goudotianus (Tul.) Skorupa; P. racemosus Vahl subsp. goudotianus (Tul.) Skorupa var. mollis (Cuatrec.) Skorupa; P. racemosus Vahl subsp. goudotianus (Tul.) Skorupa var. heterochromus (Kaastra) Skorupa. Informações sobre a distribuição geográfica, hábitat e fenologia são fornecidos para estes táxons.

Palavras-chave - Pilocarpus, Rutaceae, taxonomia

\section{Introdução}

Pilocarpus Vahl (Rutaceae) é um gênero neotropical de plantas arbustivas e arbóreas, com distribuição geográfica que se estende desde o sul do México e América Central - incluindo as Pequenas e Grandes Antilhas - até o sul da América do Sul. Skorupa (1996) reconheceu 16 espécies para o gênero, incluindo quatro novos táxons específicos (Skorupa 1998, 1999). A maioria de seus representantes ocorre no Brasil, predominantemente em sua porção oriental onde são encontradas 12 espécies, constituindo o centro de diversidade genética do gênero.

O propósito deste trabalho é apresentar novos táxons infraespecíficos e novas combinações, em antecipação à publicação da revisão taxonômica do gênero.

\section{Material e métodos}

O estudo taxonômico do qual deriva este trabalho (Skorupa 1996) foi baseado na análise de caracteres vegetativos e reprodutivos de materiais botânicos depositados nos seguintes herbários: BHCB, BM, BR, C, CAY, CEN, CEPEC,

\footnotetext{
1. Parte da Tese de Doutorado do autor, no Departamento de Botânica da Universidade de São Paulo, SP, Brasil.

2. Embrapa Meio Ambiente, Caixa Postal 69, 13820-000 Jaguariúna, SP, Brasil.skorupa@cnpma.embrapa.br.
}

CPAP, EAC, EAN, F, FUEL, G, GH, GUA, HRB, HUM, IAC, IAN, ICN, INPA, K, L, MG, MICH, MO, NY, P, R, RB, RFA, S, SP, SPF, TEPB, UEC, UPCB, US.

\section{Resultados e Discussão}

1. Pilocarpus grandiflorus Engl. in Martius \& Eichler, Fl. bras. 12(2):137, tab. 31, fig. 1. 1874. Tipo: BRASIL: Inter Vitoria et Bahia, Sello s.n. (lectótipo, aqui designado, P, fragmentos Herb. Baillon ex B). Epítipo, aqui designado: tab. 31, fig. 1, de Engler in Martius \& Eichler, Fl. bras. 12(2). 1874.

Figuras 1-18.

Engler (1874) baseou a descrição de $P$. grandiflorus em Sello s.n., único material relacionado. Sua descrição é acompanhada de uma ilustração, com análise. O material tipo depositado em $\mathrm{B}$ foi destruído. Fragmentos desse material estão depositados em P. Estes fragmentos, juntamente com a ilustração na Flora Brasiliensis, constituem os únicos materiais originais remanescentes. Kaastra (1982) não se posicionou com relação à escolha de um lectótipo. Diante disso, designa-se aqui os fragmentos do tipo em $\mathrm{P}$ como lectótipo. Por outro lado, considerando o estado precário em que se encontram esses fragmentos em $\mathrm{P}$, os quais são insuficientes para a aplicação precisa do nome do táxon, designa-se aqui como epitipo a ótima ilustração do táxon contida na Flora Brasiliensis. 
Adicionalmente à variedade típica (figuras 1-9), reconhece-se aqui uma segunda variedade, denominada var. recurvus.

1.a. Pilocarpus grandiflorus Engl. var. recurvus Skorupa var. nov. Tipo: BRASIL: BAHIA: Ilhéus, 5,5 km SW Olivença, rumo a Vila Brasil, $14^{\circ} 55^{\prime} \mathrm{S}, 3^{\circ} 07^{\prime} \mathrm{W}$, 3-IV-1992, L. A. Skorupa \& A. Amorin 992 (holótipo, CEN).

Figuras 10-18.

Bracteolae 2, oppositae, semper ad pedicellorum basin sitae, antherae lobis inferioribus et superioribus thecarum fortiter recurvatis.

Bractéolas 2, opostas, inseridas na base dos pedicelos; anteras com lobos inferiores e superiores das tecas fortemente recurvados e em contato com a porção dorsal.

Distribuição e fenologia: variedade conhecida apenas das proximidades de Olivença, sudeste da Bahia, Brasil, em florestas úmidas. Espécimes com flores e frutos coletados em fevereiro e março.

Parátipos: BRASIL: BAHIA: Ilhéus, $5 \mathrm{~km} \mathrm{SW} \mathrm{de}$
Olivença, rumo a Vila Brasil, 8-II-1982, L.A. Mattos et al. 1490 (SPF); $4 \mathrm{~km} \mathrm{SW}$ de Olivença, rumo a Vila Brasil, 18-III-1994, L.A. Skorupa 1014 (CEN); 5,4 km de Olivença, rumo a Maruim, $14^{\circ} 15^{\prime} \mathrm{S}, 39^{\circ} 02^{\prime} \mathrm{W}$, 5-II-1994, W.W. Thomas et al. 10281 (SPF).

A variedade recurvus difere da var. grandiflorus por apresentar anteras com lobos das tecas fortemente recurvados (figuras 12,14) e bractéolas opostas, sempre inseridas na base do pedicelo (figura 11); a var. grandiflorus apresenta anteras com ápices apenas levemente recurvados (figura 5) e bractéolas alternas ou subopostas, inseridas próximo ao cálice ou até a porção média do pedicelo (figura 3).

2. Pilocarpus pauciflorus A. St.-Hil., Bull. Sci. Soc. Philom. Paris, sér. 3, 10: 131. 1823. Tipo: BRASIL: SANTA CATARINA: Itapacoroia, 1816-1821, A.F.C.P. SaintHilaire s.n. (lectótipo, P; foto do lectótipo, P; isolectótipos, MPU, P).

Figuras 19-27.

Adicionalmente à subespécie típica, é reconhecida uma nova subespécie, denominada subsp. clavatus.

Chave para as subespécies de $P$. pauciflorus

1. Racemo 8,0-29,0 mm larg., corola 7,5-9,0 mm diâm; pétalas 3,0-3,7 × 1,6-2,4 mm, fortemente reflexas na antese; estigma capitado; folhas pouco reticuladas subsp. pauciflorus

1. Racemo 25,0-34,0 mm larg., corola 9,0-10,0 mm diâm.; pétalas 3,8-4,3 × 2,1-2,4 mm, pouco reflexas na antese; estigma clavado; folhas marcadamente reticuladas subsp. clavatus

2.a. Pilocarpus pauciflorus A. St.-Hil. subsp. clavatus Skorupa subsp. nov. Tipo: BRASIL: BAHIA: Itapetinga, Sítio Amarelina, ca. $5 \mathrm{~km}$ de Itapetinga rumo a Itororó, 2-IV-1992, L.A. Skorupa et al. 991 (holótipo, CEN; isótipos, NY, RB, SPF).

Figuras 23-27.

Folia dense reticulata; raceme $25,0-34,0 \mathrm{~mm}$ lati; pedicelli 5,0-17,0 mm longi; corolla 9,0$10,0 \mathrm{~mm}$ diam., petala 3,8-4,3 $\mathrm{mm}$ longa et 2,1-2,4 mm lata, paulo sub anthesi reflexa; stygmata clavata; mericarpia angulosa apice subtruncata.
Árvores até $10,0 \mathrm{~m}$ alt.; fuste cinéreo, 12,0-15,0 cm diâm. Folhas simples, alternas, subopostas ou subverticiladas, lâmina 2,2-8,0 × 1,0-3,2 cm, obovada a estreitamente obovada, estreitamente elíptica a elíptica, cartácea, densamente reticulada, nervuras secundárias 8-10 pares; pecíolo 2,0-8,0 $\mathrm{mm}$. Racemo 7,0-17,5 $\mathrm{cm} \times 2,5-3,4 \mathrm{~cm}$ na antese, raque ca. 1,5 mm diâm. na base, diminutamente pubescente, tricomas hialinos ou ferrugíneos; pedicelos $(5,0-) 13,0$ $17,0 \mathrm{~mm}$ compr., pubescentes; bráctea floral 1, 1,0$1,4 \times 0,5-0,8 \mathrm{~mm}$, triangular, ápice subacuminado, face

Figures 1-9. Pilocarpus grandiflorus var. grandiflorus. 1. Leaf. 2. Inflorescence in bud. 3. Bud. 4. Flower without petals and stamens, lateral view. 5. Stamens, dorsal, lateral and frontal view. Figures 10-18. P. grandiflorus var. recurvus. 10. Inflorescence in bud. 11. Bud. 12. Flower, oblique view without one stamen. 13. Flower, lateral view without petals and stamens. 14. Stamens, frontal, lateral and dorsal view showing anther-lobes strongly recurved. 15. Rachis with mericarps. 16. Mericarp, lateral view. 17-18. Seed, lateral and frontal view (1 - Mattos 1490; 2-3, 6-9 - Pirani 1113I; 4-5 - Sucre 8335; 10-11 - Skorupa 992; $12-18$ Skorupa 1014). 


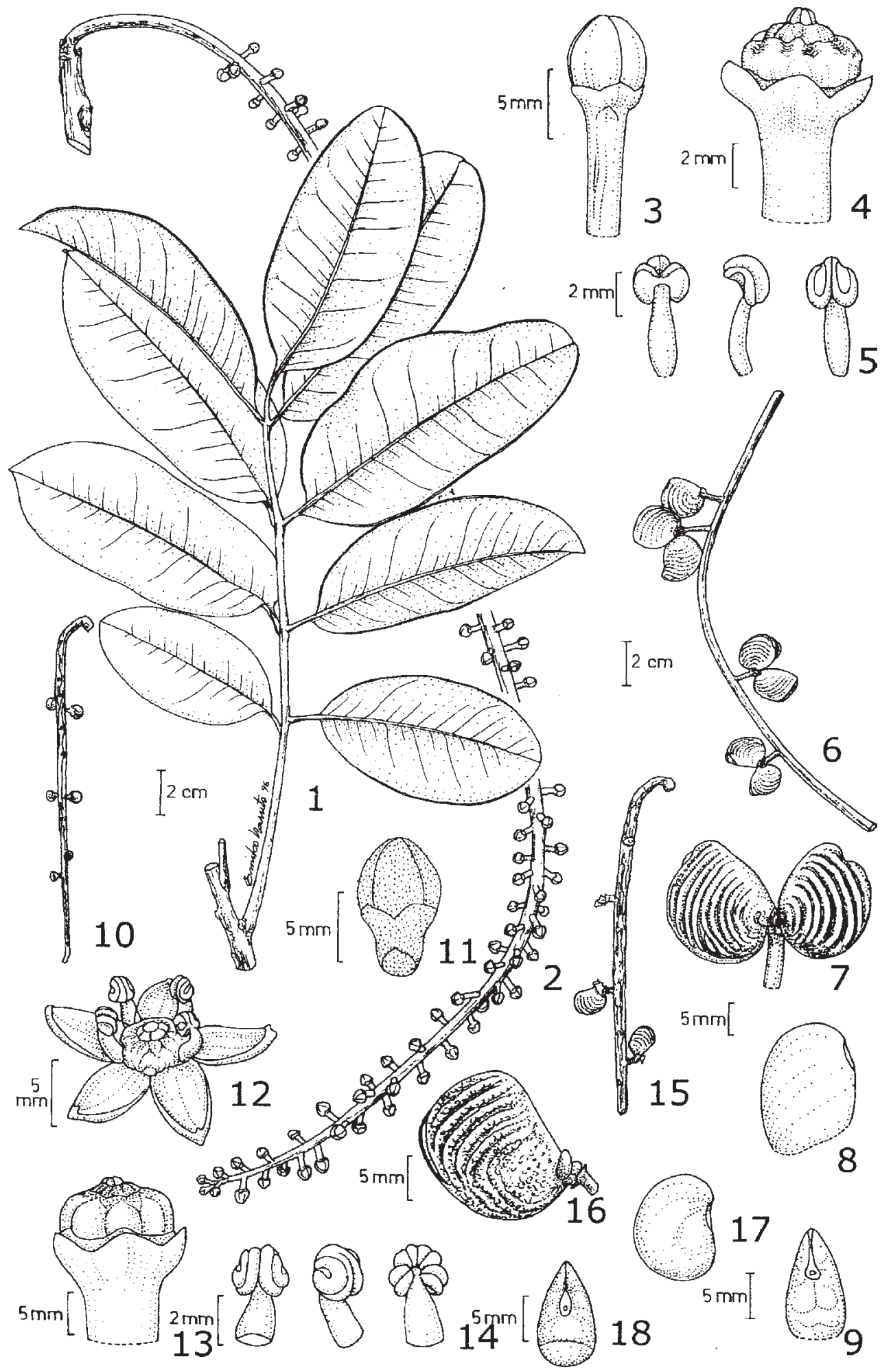

Figuras 1-9. Pilocarpus grandiflorus var. grandiflorus. 1. Folha. 2. Inflorescência em botão. 3. Botão. 4. Flor sem pétalas e estames, vista lateral. 5. Estame, vistas dorsal, lateral e frontal. 6. Detalhe da raque com mericarpos. 7. Mericarpos, vista lateral. 8-9. Semente, vistas lateral e frontal. Figuras 10-18. P. grandiflorus var. recurvus. 10. Inflorescência em botão. 11. Botão. 12. Flor, vista oblíqua sem um estame. 13. Flor, vista lateral sem pétalas e estames. 14. Estame, vistas frontal, lateral e dorsal mostrando lobos das tecas fortemente recurvados. 15. Raque com mericarpos. 16. Mericarpo, vista lateral. 17-18. Semente, vistas lateral e frontal (1 - Mattos 1490; 2-3, 6-9 - Pirani 1113; 4-5 - Sucre 8335; 10-11 - Skorupa 992; $12-18$ Skorupa 1014). 


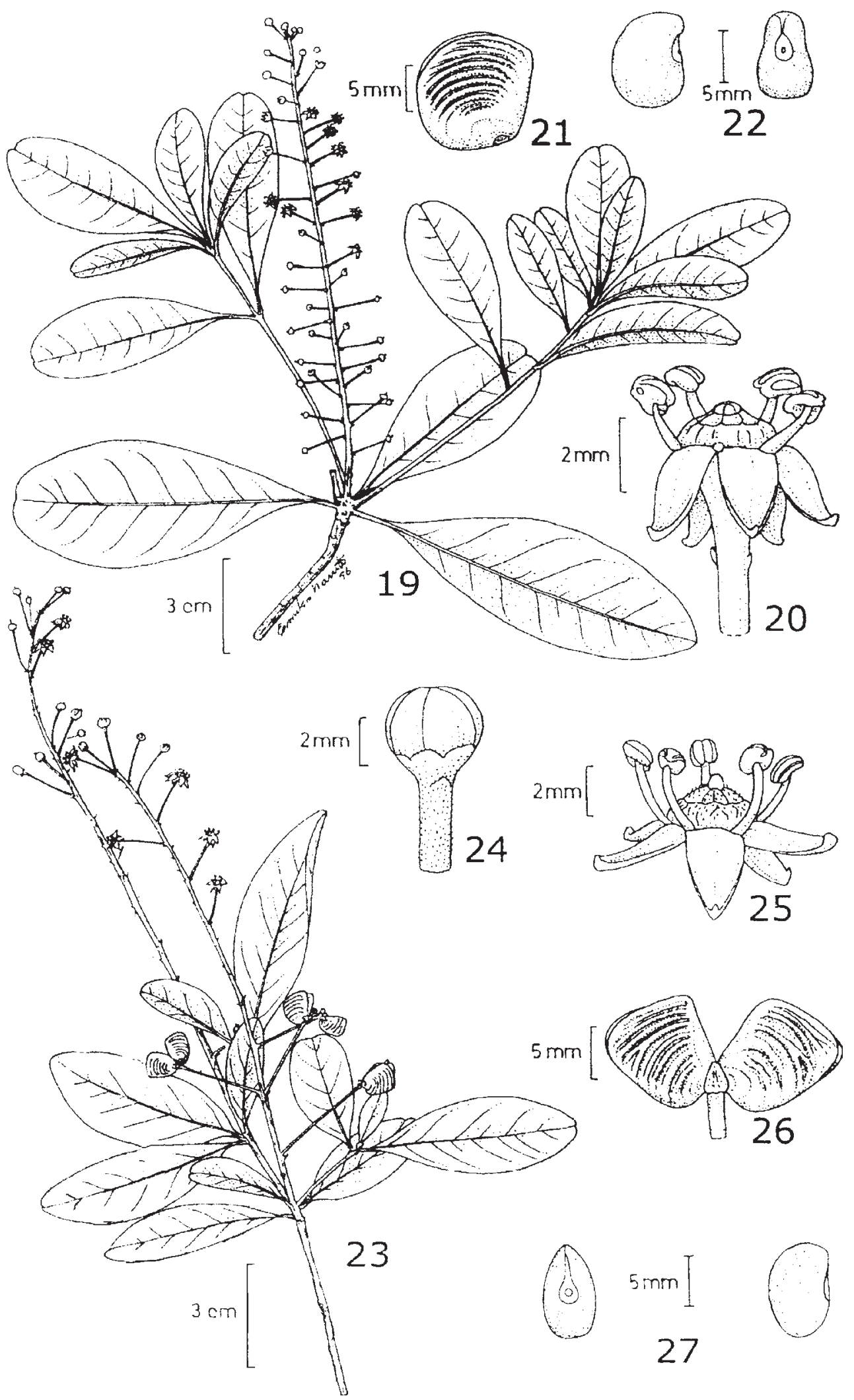

Figuras 19-22. Pilocarpus pauciflorus subsp. pauciflorus. 19. Ramo com inflorescência. 20. Flor sem um estame, vista lateral. 21. Mericarpo, vista lateral. 22. Semente, vistas lateral e ventral. Figuras 23-27. P. pauciflorus subsp. clavatus. 23. Ramo com flores e mericarpos. 24. Botão. 25. Flor, vista lateral. 26. Mericarpos, vista lateral. 27. Semente, vistas ventral e lateral (19 Catharino 790; 20-22 - Britez 753; 23-27 - Skorupa 991). 
externa estrigilosa; cálice 5-lobado, lobos ca. $1,0 \times 1,3 \mathrm{~mm}$, ovados, externamente pubérulos; corola 9,0-10,0 mm diâm.; pétalas 5, 3,8-4,3 × 2,1-2,4 mm, lanceoladas a estreitamente ovadas, face externa pubérula ou glabra, levemente reflexa na antese; filetes 3,4-3,7 $\times$ ca. $0,4 \mathrm{~mm}$, glabros, anteras 1,3-1,6 $\times$ 1,0-1,1 mm; disco ca. 2,7 mm diâm., pubescente, tricomas ferrugíneos; estigma clavado, ca. $0,6 \times 0,5 \mathrm{~mm}$. Mericarpos 10,0-11,5 × 9,0-9,5 mm, obovóides, angulados, subtruncados no ápice, linha de deiscência até $1 / 3$ da distância até o ápice; pedicelos 17,0-28,0 mm compr.; sementes 9,0-9,5 × 5,0-5,5 mm, elipsóides; hilo ca. 4,4 × 1,4-1,6 mm, estreitamente ovado.

Distribuição e fenologia: Sul da Bahia, Brasil, em florestas semidecíduas. Espécimes com flores e frutos coletados em abril.

Parátipo: BRASIL: BAHIA: Fazenda Barra da Nega, km 6 da BR 415, trecho Itapetinga-Itororó, 19-IV-1978, L.A. Mattos et al. 178 (SPF).

Pilocarpus pauciflorus é uma espécie facilmente reconhecível pela largura de suas inflorescências na antese, geralmente superior quando comparada com a das demais espécies do gênero ocorrentes no Brasil. Outras características dizem respeito à forma de suas lâminas foliares geralmente obovadas a estreitamente obovadas, com ápices obtusos a arredondados, emarginados; suas pétalas são fortemente reflexas na antese (figura 20); seus filetes são truncados e o estigma capitado. A subsp. clavatus se diferencia da subespécie típica por apresentar lâmina foliar conspicuamente reticulada, flores com pétalas apenas levemente reflexas na antese e estigma clavado (figura 25); suas peças florais também possuem dimensões geralmente superiores, como pode ser verificado na chave abaixo. Além das diferenças morfológicas apontadas acima, as duas subespécies possuem distribuições geográficas alopátricas: a subsp. clavatus ocorre apenas na Bahia, enquanto a subsp. pauciflorus, em Santa Catarina, Paraná, São Paulo, Rio de Janeiro e Espírito Santo. As variações morfológicas e o isolamento geográfico determinaram a escolha da categoria subespécie para o novo táxon.

3. Pilocarpus racemosus Vahl, Eclog. 1:29, tab. 10. 1797. Tipo: WEST INDIES: Leeward Islands, Montserrat, Ryan s.n. (holótipo, C; isótipos, BM, C, P, fotografia do isótipo $\mathrm{GH}$ ex $\mathrm{C}$ ).

Figuras 28-43.

Skorupa (1996) reconheceu três subespécies em P. racemosus: subsp. racemosus, subsp. viridulus Kaastra e subsp. goudotianus (Tul.) Skorupa, esta última como resultado de uma nova combinação.

\section{Chave para as subespécies de Pilocarpus racemosus}

1. Folhas (1-)2-3(-4)-jugadas, unifolioladas ou simples; flores com pétalas inteiramente esverdeadas

1. Folhas 1(-2)-jugadas, unifolioladas ou simples, flores com pétalas vináceas a purpúreas, ou, menos freqüentemente, esverdeadas na porção superior

2. Folhas com lâmina glabra, menos freqüentemente pubérula na base ou na face abaxial, tricomas adpressos; pecíolo glabro ou apenas pubérulo subsp. racemosus

2. Folhas com lâmina pubescente em ambas as faces ou apenas ao longo da nervura principal; pecíolo pubescente subsp. goudotianus

3.a. Pilocarpus racemosus Vahl subsp. goudotianus (Tul.) Skorupa comb. et stat. nov.

Basiônimo: Pilocarpus goudotianus Tul., Ann. Nat. Bot. Ser. 3. 7: 284. 1847. Tipo: COLÔMBIA: ToLimA: between Aipe at Rio Magdalena and Ataco, IV-1844,
Goudot s.n. (holótipo e foto do holótipo, P; isótipo, K). Figuras 37-43.

A subespécie goudotianus difere das demais subespécies de $P$. racemosus por apresentar folhas simples, unifolioladas ou 1-jugadas, assim como lâminas

Figures 19-22. Pilocarpus pauciflorus subsp. pauciflorus. 19. Branch with inflorescence. 20. Flower without one stamen, lateral view. 21. Mericarp, lateral view. 22. Seed, lateral and ventral view. Figures 23-27. P. pauciflorus subsp. clavatus. 23. Branch with flowers and mericarps. 24. Bud. 25. Flower, lateral view. 26. Mericarps, lateral view. 27. Seed, ventral and lateral view (19 - Catharino 790; 20-22 - Britez 753; 23-27 - Skorupa 991). 


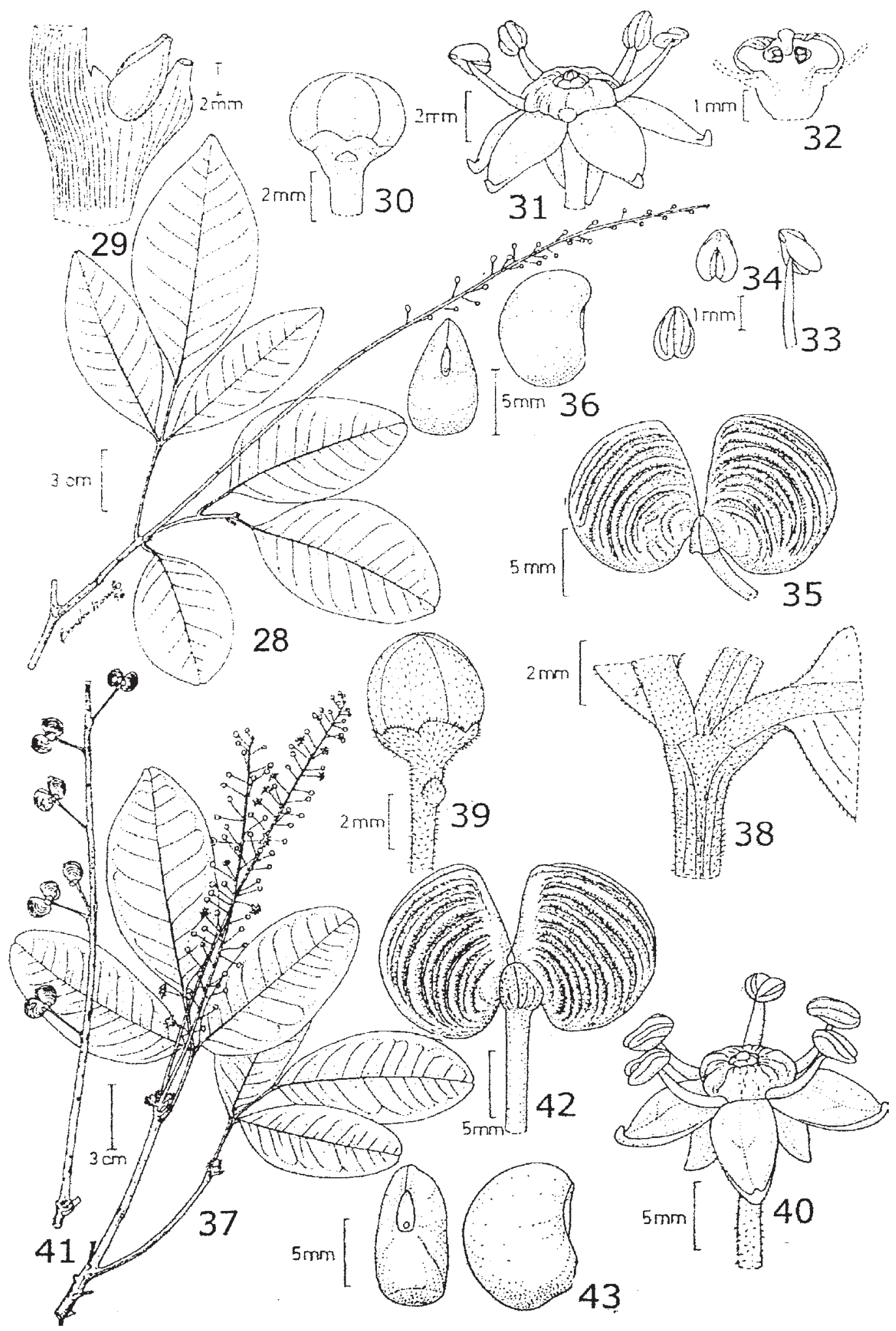

Figuras 28-36. Pilocarpus racemosus subsp. racemosus. 28. Ramo com inflorescência. 29. Catáfilos glabros. 30. Botão floral. 31. Flor sem um estame, vista oblíqua. 32. Ovário em corte longitudinal. 33. Estame, vista dorsal. 34. Anteras, vistas dorsal e frontal. 35. Mericarpos, vista lateral. 36. Semente, vistas ventral e lateral. Figuras 37-43. Pilocarpus racemosus subsp. goudotianus. 37. Ramo com inflorescência. 38. Detalhe da pilosidade do pecíolo, peciólulo e nervura central. 39. Botão floral. 40. Flor, vista oblíqua. 41. Raque com mericarpos. 42. Mericarpos, vista lateral. 43. Semente, vistas ventral e lateral (28-29, 35-36 - Duss 1193; 30-34 - Herrera 1521; 37-40 - Xena 591; 41-43 - Sobel 2067). 
foliares pubescentes; a subsp. racemosus possui folhas simples, unifolioladas ou até 2-jugadas e lâminas foliares essencialmente glabras; a subsp. viridulus, por sua vez, apresenta folhas simples, unifolioladas ou até 4-jugadas, lâminas foliares glabras e flores completamente esverdeadas, sem a coloração vinácea a purpúrea observada nas demais subespécies de $P$. racemosus. As três subespécies apresentam morfologia floral muito similar e também bastante peculiar quando comparada com a observada nas demais espécies do gênero. Entre as características peculiares apresentadas conjuntamente apenas por esses três táxons, ressalta-se a ocorrência de um disco bem desenvolvido, envolvendo a base dos filetes; filetes conspicuamente subulados com ápice agudo e estigma clavado (figuras 31, 33, 40). Além dessas características, seus frutos e sementes são bastante similares em tamanho, forma e cores (figuras 35, 36, 42, 43). Kaastra (1982) revelou sua intenção de reunir $P$. goudotianus Tul. e $P$. racemosus Vahl em um único táxon, reconhecendo uma grande proximidade dos dois táxons com base em suas características morfológicas. Isso acarretaria a formação de um táxon com demasiados táxons infraespecíficos, argumento que o leva a mantê-los separados. Skorupa (1996) concluiu que se justifica a acomodação de $P$. goudotianus como uma subespécie de $P$. racemosus, dadas as afinidades morfológicas apresentadas acima, somadas aos distintos padrões de distribuição geográfica apresentados pelas subespécies: a subsp. goudotianus ocorrendo ao norte e oeste da Colômbia e noroeste da Venezuela e a subsp. racemosus com uma distribuição mais ampla, ocorrendo no México, Pequenas e Grandes Antilhas, noroeste da Colômbia e noroeste da Venezuela. A subsp. viridulus está restrita à Costa Rica e El Salvador.

Kaastra (1982) reconheceu a subsp. heterochromus em P. goudotianus Tul., apresentando as seguintes características discriminadoras: flores com pétalas e filetes purpúreos na base e esverdeados nas porções superiores, segmentos do cálice dentados, catáfilos glabros e pecíolos glabrescentes. Considerando tratar-se de variações que não alteram a conceituação da subsp. goudotianus, como aqui considerada, propõese a redução da subsp. heterochromus à condição de variedade de $P$. racemosus subsp. goudotianus, nova combinação apresentada acima. Desta forma, como conseqüência dessa nova combinação, surgem outras novas combinações ao nível das variedades, a saber: subsp. goudotianus var. goudotianus; subsp. goudotianus var. mollis, e subsp. goudotianus var. heterochromus.

Chave para as variedades de Pilocarpus racemosus subsp. goudotianus

1. Flores com pétalas e filetes esverdeados, ou esverdeados na porção superior e purpúreos em direção à base; catáfilos glabros protegendo as gemas var. heterochromus

1. Flores com pétalas e filetes inteiramente vináceos a purpúreos; catáfilos pilosos protegendo as gemas

2. Lâminas subcoriáceas a coriáceas, pubescentes a glabrescentes; racemos 13,0-40,0 mm larg., pedicelos 5,0-20,0 $\mathrm{mm}$ compr. var. goudotianus

2. Lâminas cartáceas, intensamente pubescentes em ambas as faces; racemos 48,0-50,0 mm larg., pedicelos 15,0-26,0 mm compr. var. mollis

Figures 28-36. Pilocarpus racemosus subsp. racemosus. 28. Branch with inflorescence. 29. Cataphylls glabrous. 30. Bud. 31. Flower without one stamen, oblique view. 32. Ovary in longitudinal section. 33. Stamen, dorsal view. 34. Anthers, dorsal and frontal view. 35. Mericarps, lateral view. 36. Seed, ventral and lateral view. Figures 37-43. Pilocarpus racemosus subsp. goudotianus. 37. Branch with inflorescence. 38. Detail of the pilosity of the petiole and midvein. 39. Bud. 40. Flower, oblique view. 41. Raquis with mericarps. 42. Mericarps, lateral view. 43. Seed, ventral and lateral view. (28-29, 35-36 - Duss 1193; 30-34 - Herrera 1521; 37-40 - Xena 591; 41-43 - Sobel 2067). 
3.a.1. Pilocarpus racemosus Vahl subsp. goudotianus (Tul.) Skorupa var. heterochromus (Kaastra) Skorupa, comb. et stat. nov.

Basiônimo: Pilocarpus goudotianus Tul. subsp. heterochromus Kaastra, Acta Bot. Neerl. 26:486. 1977. Tipo: COLÔMBIA: HuILA: upper basin of Rio Magdalena, Cabrera Lajas, ca. $11 \mathrm{~km}$ E of Villavieja on road to Baraya, 4-VII-1950, S.G. Smith 1136 (holótipo, $\mathrm{UC}$; isótipos, $\mathrm{F}, \mathrm{GH}, \mathrm{MO}$, US).

A var. heterochromus é conhecida apenas da localidade tipo, no centro-oeste da Colômbia.

3.a.2. Pilocarpus racemosus Vahl subsp. goudotianus (Tul.) Skorupa var. mollis (Cuatrec.) Skorupa comb. nov. Basiônimo: Pilocarpus alvaradoi Pittier var. mollis Cuatrec., Rev. Acad. Colomb. Ci. Exact. y Nat. 8:467. 1952. Pilocarpus goudotianus Tul. subsp. goudotianus var. mollis (Cuatrec.) Kaastra, Acta Bot. Neerl. 26:486. 1977. Tipo: COLÔMBIA: MagdalenA: forest along highway, $70 \mathrm{~km}$ southeast of Riohacha, 80m, 7-XII-1944, O. Haught 4480 (holótipo, US; isótipos, COL, GH, K, NY, UC). Syn. nov.
A var. mollis é conhecida apenas da localidade tipo, a noroeste da Colômbia.

Agradecimentos - $\mathrm{O}$ autor agradece a Tarciso S. Filgueiras pelas diagnoses em Latim e a Emiko Naruto pelas ilustrações.

\section{Referências bibliográficas}

ENGLER, A. 1874. Rutaceae: Pilocarpus. In Flora brasiliensis (C.F.P. Martius \& A.G. Eichler, eds.). R. Oldenbourg, Munich \& Leipzig, v.12, p.131-139.

KAASTRA, R. 1982. Flora neotropica-monograph 33. Pilocarpinae. New York Botanical Garden, New York.

SKORUPA, L.A. 1996. Revisão taxonômica de Pilocarpus Vahl (Rutaceae). Tese de doutorado, Universidade de São Paulo, São Paulo.

SKORUPA, L.A. 1998. Three new species of Pilocarpus Vahl (Rutaceae) from Brazil. Novon 8:447-454.

SKORUPA, L.A. 1999. A new species of Pilocarpus Vahl (Rutaceae) from Peru. Novon 9:257-259. 